\title{
Mirar con nuevos ojos: las buenas prácticas en contextos desfavorables*
}

Estela Serventich**

"El verdadero viaje al descubrimiento no consiste en ver nuevos paisajes sino en tener nuevas miradas".

Marcel Proust

\begin{abstract}
In this article we present the characteristics of good practices of education that manage to excite young people of unfavorable contexts, to promote their interest on knowledge and actual participation in the learning processes.

From the analysis of the diverse sources it arises that the search of understanding from the aspect of epistemology and moral underlies these practices. The different educational devices are characterized by the flexibility demonstrated in the method adopted for class dialogue, the attention to emergent issues, the way errores are treated, the search of collaborative work and time management. They are based on a personal and professional commitment, framed into the ethical professionalism that comes from assuming the work with these students as a challenge , challenge that arises from the encounter with a new social reality from which new student populations emerge, characterized like "poor" in teacher speech.
\end{abstract}

\section{Resumen}

En este artículo presentamos las características de buenas prácticas de enseñanza que logran entusiasmar a los jóvenes de contextos desfavorables, propiciando el interés por el conocimiento y la participación real en los procesos de aprendizaje.

Del análisis de las diversas fuentes surge que subyace a esas prácticas la búsqueda de la comprensividad desde las vertientes epistemológica y moral. Los diferentes dispositivos de enseñanza se caracterizan por la flexibilidad evidenciada en la forma que adopta el diálogo en clase, la atención a temas emergentes, el modo de tratar el error, la búsqueda del trabajo colaborativo y el manejo de los tiempos. Se fundamentan en un compromiso personal y profesional docente, enmarcado en la profesionalidad ética que deriva de asumir como desafío el trabajo con estas poblaciones, desafío que surge del encuentro con una realidad social nueva de la que emergen poblaciones estudiantiles nuevas, caracterizadas como pobres en los discursos docentes.

\section{De Poros y Aporías, problema y objetivos}

El presente artículo es una síntesis del informe de tesis presentado en el marco de la Maestría en Educación en el año 2008. La investigación se realizó durante el año 2007 en dos liceos de Ciclo Básico de la zona noroeste de Montevideo, teniendo entre sus objetivos la comprensión del sentido que adquieren las buenas prácticas de enseñanza en contextos desfavorables. 
El tema elegido es producto de intereses y conocimientos personales y circunstancias históricopolítico-económicas e institucionales actuales, reconociéndolo como uno de centros de preocupación de las comunidades de investigación actual. Por otra parte, la inclusión social y educativa es un discurso emergente.

Consideramos pertinente remitirnos a la noción de problema desde la etimología griega de aporía. Poros significa "paso, puente, camino, calle", aquello que permite el paso. Aporos remite a "intransitable, sin camino o salida, difícil"; aquello que obstaculiza o impide el paso; también es "necesitado, pobre". Poros es la riqueza, aquello que no presenta obstáculos. Pensar problemas supone pensar desde una carencia que reconoce la presencia de un obstáculo que dificulta el pasaje. Por tanto, el primer paso supone hacer visible el obstáculo, problematizar. Un segundo paso consistiría en buscar los modos de superar la aporía, esto es, lograr pasar al otro lado del obstáculo ${ }^{1}$.

En nuestro trabajo reconocimos como un obstáculo la situación actual de los centros de Ciclo Básico de Enseñanza Secundaria Pública de la zona NW de Montevideo cuya población se conforma por sectores sociales pertenecientes a contextos desfavorables caracterizada por: baja retención y bajos rendimientos académicos, un cuerpo docente que requiere actualización en lo técnico pedagógico y sensibilización ante el contexto socio-cultural y económico en que trabajan.

Ante esta situación que obstaculiza el "paso" nos planteamos como problema:

- ¿Existen prácticas de enseñanza que logren entusiasmar a los jóvenes de contextos desfavorables, propiciando el interés por el conocimiento y la participación real en los procesos de aprendizaje?

- Si existen ¿qué características tienen? y ¿cuáles son los dispositivos de enseñanza puestos en juego que tienen impacto en esas poblaciones?

- ¿Cómo se vinculan estas prácticas con la historia personal y profesional de los docentes?

Delimitado el problema nos propusimos como objetivos:

- Comprender a través de la observación de clase en condiciones naturales, el sentido que adquieren las buenas prácticas de enseñanza en contextos desfavorables.

- Indagar las relaciones entre la historia personal y profesional de los docentes que trabajan en contextos desfavorables.

- Construir aproximaciones teóricas que contribuyan al conocimiento y comprensión de esas prácticas.

\section{La relevancia del tema}

En las últimas décadas, se produjo una expansión matricular en la enseñanza media que permitió acceder a la misma a miles de jóvenes de contexto desfavorable, lo que conlleva un efecto democratizador, pero ha traído consecuencias. Por otra parte, en los años 2000, la expansión de la pobreza y la exclusión social ha conducido a los centros y a los docentes a asumir funciones de contención social y afectiva, en menoscabando de su su función pedagógica, recayendo sobre la escuela demandas que la trascienden.

Distintos informes e investigaciones (MEMFOD-ANEP, 2003; UNICEF, 2003; MESyFOD-ANEP - UTU/BID 2000; MESyFOD-ANEP,1999; ANEP- UMNRE, 1999) dan cuenta del descenso de los niveles de rendimiento de los estudiantes y el aumento de la repetición y deserción; el fracaso escolar es el resultado de múltiples condiciones sociales, institucionales e individuales que interactúan con diferente incidencia, siendo más compleja la interacción entre las condiciones socio económicas desfavorables y los rendimientos en los aprendizajes de los jóvenes liceales. 
Las investigaciones ya citadas nos dicen que cuanto mayor es la distancia cultural entre el centro educativo y el hogar, más se requiere la intervención pedagógica desde el centro y desde el aula de modo tal que las prácticas consideren las características contextuales de cada centro, a través de propuestas que articulen lo que el alumno ya trae (cultura, lenguaje, experiencia y conocimientos) con lo que se pretende que logre. Cuando la escuela se abre a todos los sectores sociales, las diferencias en el origen y en las expectativas crean diferentes posicionamientos frente a las instituciones que requieren nuevos mecanismos de integración.

Subyace a esta problemática la noción de inclusión social (Pedernera, 2005; Laurnaga, 2005; Gentili, 2001; Rivero, 1999) que contiene en forma implícita la de exclusión social, entrañando una tensión dinámica ya que por el propio contenido se define por el opuesto: el adentro y el afuera, constatando la exclusión de, se define la necesidad de la inclusión en.

Con relación a los factores pedagógicos que inciden en el aprendizaje - fracaso escolar (MESyFOD- ANEP-UTU/BID, 2000; ANEP-UMRE, 1999; Fernández, 1996; Ravela, 1993) se encuentran aspectos tales como la experiencia del docente, la estabilidad en el centro educativo, el compromiso con el centro, el conocimiento del medio, sus expectativas, la satisfacción con la tarea, la actualización pero no encontramos referencia específica a las características de las buenas prácticas de enseñanza en contextos desfavorables. En nuestro actual contexto sociopolítico signado por la preocupación generalizada por los resultados de la enseñanza, conviene señalar la oportunidad de este trabajo que procura compartir hallazgos y reflexiones sobre las características de las buenas prácticas de enseñanza en esos contextos.

\section{Líneas teóricas de partida}

Actualmente existe la preocupación y la necesidad de promover prácticas que hagan de los centros educativos de enseñanza media un lugar de acogida que propicie la inclusión de los jóvenes de contextos desfavorables, que sean "una puerta de entrada a la cultura (Bruner, 1997)". Abordar este problema implica conceptualizar buena enseñanza, buenas prácticas de enseñanza y pobreza, ésta referida en particular a la problemática del contexto y su relación con el sistema educativo uruguayo.

\section{Buena enseñanza}

La enseñanza es una actividad que supone que unas personas ejerzan influencias sobre otras en relación de desigualdad de poder y autoridad (Contreras, 1994). Responde a una intencionalidad educativa, llevándose a cabo actividades que se justifican por su valor con respecto a fines deseables. No se puede ser indiferente ante el tipo de interacción que se establece entre profesor y alumnos, ni ante lo que se les pretende enseñar y cómo. Si la enseñanza es una actividad intencional que obliga moralmente a sus responsables, también es una práctica social que excede a su comprensión como producto de decisiones individuales, generando una dinámica que sólo puede comprenderse en el marco del funcionamiento general de la estructura social de la que forma parte.

Entre las tareas de enseñanza y las tareas de aprendizaje existe una relación de dependencia ontológica, mediada por el flujo de tareas que establece el contexto institucional y dentro del cual se descubre el modo de realización de las tareas de aprendizaje. Son éstas las que pueden dar lugar a aprendizajes. La comprensión de las mediaciones entre estos dos conceptos, de la dependencia, pero a la vez desigualdad y corte entre ambos, justifica el uso de un concepto más complejo que el de enseñanza para expresar el referente de la Didáctica, como es la expresión "procesos de enseñanza-aprendizaje". Entendemos por procesos de enseñanza-aprendizaje, el sistema de comunicación intencional que se produce en un marco institucional y en el que se generan estrategias encaminadas a provocar el aprendizaje. 
Podemos pensar en la enseñanza como la elaboración de estrategias para entender y acortar las distancias entre las condiciones de realidad y las aspiraciones educativas. Esto nos remite a considerar el papel que desempeña el profesor, en cuanto figura fundamental en el desarrollo de la estrategia de enseñanza. Es él quien tiene que comprender el funcionamiento de lo real y articular su visión crítica de esa realidad con sus pretensiones educativas, que a su vez se definen y reformulan en función de los contextos específicos y de las experiencias acumuladas. Esto significa definir el trabajo del profesor como intelectual y no como técnico. Jackson (2002) plantea qué deben saber los docentes sobre la enseñanza, si existe algo más que la pericia técnica y nos remite a los requerimientos epistémicos de la enseñanza como método y la necesidad del docente de dominar el material que enseña. Asimismo la propia biografía o lo que él llama el "sentido escolar", brinda una idea al sujeto de lo que implica la tarea docente en cuanto a conocimientos y aptitudes, pero no es suficiente, como tampoco lo es el sentido común, ya que no siempre habla con una sola voz. Hace falta el buen sentido.

El análisis de los criterios que empleamos para determinar qué es lo que se considera buena enseñanza, no implica el uso del adjetivo "buena" como un sinónimo de "con éxito". Fenstermacher (1989) entiende por buena enseñanza aquellas acciones que se basan en principios morales "(...) y son capaces de provocar acciones de principio por parte de los estudiantes" y poseen también fuerza epistemológica. Buen sentido, buenas intenciones, buenas razones, buenas prácticas. No hay una sola versión de buena enseñanza. La buena enseñanza no implica una única manera de actuar, sino muchas.

La definición de buena enseñanza implica la recuperación de la ética y los valores en las prácticas de enseñanza. La enseñanza es un proceso de construcción cooperativa y por ello, los alcances del pensamiento reflexivo y crítico se dan en el aula con los sujetos implicados. La buena enseñanza, por tanto, sería la expresión de una práctica reflexiva y moral y es un constructo de la didáctica actual que da cuenta de las perspectivas que enmarcan el pensamiento pedagógico contemporáneo (Litwin, 1997). El desafío actual consiste en hallar alternativas para los problemas que enfrentan los docentes en las aulas e interpretar nuestras intervenciones en el contexto de la buena enseñanza. En el campo de la didáctica implica la búsqueda de dimensiones de análisis que den cuenta de las características de la buena enseñanza.

\section{Buenas prácticas de enseñanza}

Usaremos prácticas de enseñanza en el sentido de dispositivo de intervención o acciones con sentido construido ${ }^{2}$. Entendemos por buenas prácticas de enseñanza las que favorecen "la construcción del conocimiento de los alumnos" (Litwin, 1997: 11-12), aquellas que logran entusiasmar a los jóvenes o clases reflexivas o que se reconocen como creativas y que dan sentido al esfuerzo del alumnado por aprender.

El contexto en el que se inscriben las prácticas educativas y las condiciones que las enmarcan, conforman el ámbito de significación de las mismas, ya que como señala Popkewitz (1986) establecer vínculos entre el saber y las instituciones en diferentes encuadres históricos posibilitaría comprender las prácticas educativas no como fenómenos individuales sino como prácticas sociales. La complejidad del contexto lleva al docente a diseñar estrategias que favorezcan procesos cognitivos en el estudiante con el fin de generar la construcción del conocimiento. Las actividades de enseñanza tienen lugar dentro de una serie de contextos, "entornos" que definen el medio en el cual se produce la enseñanza: individual, de grupo, la clase, la escuela, la comunidad y en cada uno de esos niveles anidados se producen las transacciones que comprenden la vida en el aula (Shulman, en Wittrock comp., 1989: 20). 


\section{Pobreza}

Los factores más relevantes que caracterizan la sociedad uruguaya actual son: la pobreza, la exclusión sociolaboral y el aumento de la desigualdad social.

Con respecto a la pobreza, Pedernera (2005: 31) sostiene que la misma es una situación de no posesión material o cultural "que no necesariamente ataca procesos de filiación y horizontes de futuro". El Programa de Naciones Unidas para el Desarrollo (PNUD) determinó tres elementos básicos para medir las condiciones de vida; se considerará a una persona o familia en situación de pobreza o no, por la conjunción de esas tres condiciones y su satisfacción. El primer elemento es el de la supervivencia (condiciones de salud y acceso al agua potable y medicinas); el segundo elemento hace a los niveles de conocimiento o acceso a la educación y el tercero refiere a los niveles de ingreso, acceso a empleos, ejercicio de los derechos políticos y a la condición de la mujer (Rivero, 1999).

Max - Neef (1984: 236) propone la diferenciación entre pobreza y pobrezas. La pobreza refiere a la situación de aquellas personas que se ubican por debajo de cierto nivel de ingresos, en tanto que las pobrezas se definen de acuerdo a necesidades no satisfechas. Las pobrezas, cuando son intensas y prolongadas, generan patologías colectivas de la frustración que necesitan de abordajes transdisciplinarios. En una línea similar, María Teresa Sirvent (1998) acuñó la expresión múltiples pobrezas haciendo referencia a una "compleja realidad de pobrezas" vinculadas a amenazas, pérdidas y miedos que fomentan nuevas formas de subjetividad. Habla también de "una pobreza de comprensión o de entendimiento" que considera "los factores sociales que nos dificultan el manejo reflexivo de la información y la construcción de un conocimiento crítico sobre nuestro entorno cotidiano".

\section{Escuela y pobreza}

El Estado moderno a través de dispositivos institucionales (escuela, familia, fábrica, hospital, cuartel, prisión) aseguraba "la producción y reproducción de su soporte subjetivo: el ciudadano" (Lewkowicz, 2004), dando a su vez sentido y consistencia a dichos dispositivos; el Estado era productor de subjetividad también desde la ley, ante la que todos los ciudadanos somos iguales, base del contrato social. A partir de los procesos de globalización se produjeron cambios a nivel estatal y en el mundo del trabajo, instaurando la sociedad de mercado, con la consecuente precarización y flexibilización laboral, dejando al sujeto librado a su suerte. El desarrollo tecnológico trajo aparejada la desindustrialización que implicó el pasaje de un modo de producción sustentado en la energía manufacturera a un sistema productivo cuyo eje es el conocimiento (Mallo, 2004), de modo tal que se equipara desarrollo económico a calidad de recursos humanos y la calidad de estos estará en función de las instituciones capaces de formarlos, entre ellos, el sistema educativo.

La escuela como institución del Estado moderno se constituyó en la principal herramienta para la formación de ciudadanos, generando identidad nacional. Sus rasgos principales fueron la homogeneidad y la inclusión por sus principios de laicidad, gratuidad y obligatoriedad. A partir de los cambios que se produjeron a nivel estatal y social, la institución escuela aparece ligada a las demandas del mercado pero perdiendo la capacidad de cumplir su función al no acompañar los diversos sentidos del cambio.

Algunos desafíos que la escuela como institución enfrenta son: la homogeneización de la población desde el punto de vista de la situación socio - económica, poblaciones empobrecidas; la ruptura del pacto escuela - familia por las propias dificultades para asumir sus funciones fundantes; las condiciones de educabilidad de los niños y adolescentes; déficits en el desarrollo motriz y cognitivo básico; subjetividades (modos de ser, estar, hacer y pensar) diferentes a las que propone. 
Desde el marco jurídico nacional e internacional, se reconoce el derecho de todo niño y adolescente a la educación y se mandata a los Estados parte, la implementación de políticas educativas que respeten este derecho en su plenitud. "La inclusión educativa es un aspecto de la inclusión en la sociedad y en ambos casos es una opción ética, de valor, cifrada en dar la bienvenida a la diferencia y apoyar la equiparación de oportunidades de los que se encuentran en desventaja." (Echeita en De Armas, 2005). La tendencia en las últimas décadas ha sido la universalización en el acceso y permanencia al sistema educativo, pero esto oculta "la existencia de redes educacionales estructuralmente diferenciadas y segmentadas" (Gentili, 2001) y no ha significado que no existan procesos de exclusión - incluyente que se desplazaron hacia el interior de la institución escolar.

Si bien Uruguay se caracterizó por niveles de equidad e integración social relativamente altos, diversos elementos y tendencias permiten identificar "nuevos clivajes sociales", que refuerzan la creciente fragmentación económica y sociocultural que comienzan a manifestarse a fines de los noventa. Filgueira (2002) identifica las siguientes transformaciones relacionadas con el agravamiento de la exclusión social: a) problemas del mercado de empleo (precarización, insuficiencia de generación de puestos, flexibilidad, etc.); b) cambio de actitudes y comportamientos en materia demográfica y de la organización familiar y c) la progresiva segmentación social a nivel residencial, educativo y laboral. Estos fenómenos se han agudizado a partir de la crisis desatada en 2002. Otros estudios (Moser, 1998; Portes, 1998) contribuyen al análisis de los contextos en que se desenvuelven las familias y las comunidades pobres. En tal sentido, en función a la tendencia creciente de los niveles de pobreza, a partir de la segunda mitad de los años noventa, surgen nuevas formas de fragmentación que implican la "formación de fronteras sociales" y la disminución de la interacción entre personas de diferente origen socio económico.

Estas transformaciones han puesto en crisis la oferta tradicional de la educación que se manifiesta a través de: exclusión, fracaso escolar, conflicto, desorden, violencia, dificultades de integración a las instituciones, ausencia de sentido de la experiencia escolar para proporciones significativas de jóvenes. Los centros educativos no han sido preparados para enfrentar este desafío por lo que surge la necesidad de repensar estrategias de apoyo desde el centro y desde el aula, teniendo en cuenta que estos grupos requieren atención pedagógica específica que los acerque e involucre con el centro educativo. Por su parte, los docentes manifiestan sentirse incapaces de desarrollar su labor con estas poblaciones y señalan la complejidad de la misma.

Por otra parte, si bien la comunidad local ejerce un papel sustantivo en el cumplimiento de normas y comportamientos aceptados socialmente es el sistema educativo, el principal ámbito capaz de crear un contexto en el cual los adolescentes pobres tengan posibilidad de mantener una relación cotidiana y desarrollar códigos comunes, vínculos de solidaridad y afecto bajo condiciones de igualdad con sus pares de otros estratos, favoreciendo el sentimiento de comunidad. El enriquecimiento del capital social será mayor cuanto mayor sea la semejanza entre la comunidad escolar de cada establecimiento y la comunidad nacional. A medida que aumenta la segmentación entre centros educativos, aumenta la posibilidad de que los miembros de un estrato social sólo se encuentren en una relación cara a cara con miembros de otros estratos sociales a nivel laboral donde tales relaciones ya estarán enmarcadas en patrones jerárquicos de la organización del mundo laboral (Katzman, 2001; 1996).

En nuestro país podemos ubicar como antecedentes distintas experiencias orientadas a dar respuesta a problemas como la repetición y la deserción, apuntando fundamentalmente a la inclusión de jóvenes pertenecientes a estratos socioeconómicos bajos, a saber: Áreas Pedagógicas (convenio Instituto del Niño y del Adolescente del Uruguay - Consejo de Enseñanza 
Secundaria), el Instituto del Hombre, la Red de Casas Jóvenes y el Programa Aulas Comunitarias de reciente implementación. A nivel internacional encontramos el Proyecto de Escuelas Aceleradas ${ }^{5}$ cuyo objetivo es la creación de escuelas que brinden oportunidades de aprendizaje enriquecidas y aceleradas a todos los alumnos durante todo el curriculum.

\section{Metodología}

La investigación se enmarcó en un modelo cualitativo; focalizamos en situaciones y personas específicas y pusimos el énfasis en las palabras de los actores a través de la observación, la narrativa de los actores y el análisis documental, permitiendo un cuadro complejo y holístico. Por otra parte, la investigación de corte cualitativo permite un estudio en profundidad con un diseño flexible; el investigador crea el método, no se limita a seguirlo. El énfasis se ubica en la generación de conocimientos.

\section{Decisiones de muestreo}

Como contexto relevante al problema de investigación seleccionamos dos liceos de la zona NW de Montevideo. Como informantes clave recurrimos a los respectivos equipos de Dirección, quienes ponderaron las prácticas de aquellos docentes que lograban entusiasmar a los jóvenes o evidenciaban la intención de favorecer la comprensión y generaban procesos de construcción del conocimiento. En base a la opinión de estos y considerando el universo (conjunto de docentes de los liceos $X$ e $Y$ ), elegimos una muestra de cuatro profesores teniendo en cuenta los criterios de accesibilidad y heterogeneidad y considerando el principio de saturación (Valles, 1999).

\section{Técnicas para la obtención de datos}

Los datos fueron obtenidos por procedimientos de observación, entrevista y análisis de documentos. Trabajamos en dos centros donde entrevistamos a sus directoras y a los docentes de la muestra observados.

Realizamos entrevistas semi-estructuradas al Equipo de Dirección para obtención de información significativa como informantes claves. Efectuamos observaciones y grabaciones de clase con la pauta de una planilla y conversación informal posterior a la observación. La observación de clase nos permitió recuperar las propuestas didácticas.

Otro instrumento usado fue la entrevista estructurada de corte fenomenológico, en base a una guía de preguntas de respuesta abierta. Este tipo de entrevista nos permitió acercarnos a la significación que los actores producen de sus propias prácticas ${ }^{3}$. Por último, efectuamos un estudio narrativo de distintos tipos de documentos: publicaciones y narrativas personales: relatos biográficos, testimonios.

Realizamos una triangulación a los efectos de la validación entre los datos surgidos del análisis documental, de las observaciones de clase, de las entrevistas realizadas a los docentes seleccionados y a los equipos directivos en carácter de informantes calificados.

El tipo de observación implementado responde a las características de la observación no participante en condiciones naturales, ya que constituye la modalidad más adecuada para este caso. Durante las mismas identificamos elementos generales relativos al clima áulico, la temática abordada, presentación del tema, contextualización, secuenciación temática, modalidades de trabajo de los diversos contenidos, estilo de conducción del docente, utilización de recursos, 
aspectos grupales (socialización, actitud de los alumno: atención, intervención espontánea, a requerimiento del profesor, preguntas formuladas). Hicimos notas de campo para organizar las observaciones, fundamentalmente notas condensadas correspondientes al desarrollo de la clase, lo cual posibilitó documentar aspectos como el uso del pizarrón o acontecimientos que ocurrían en la clase y que no se podían grabar.

Las entrevistas se dirigieron a aproximarnos a acontecimientos y actividades que no podíamos observar directamente. Elaboramos guiones procurando explorar: aspectos vinculados a la historia personal y profesional de los docentes (abarcando las tres fases de la formación docente: la biografía escolar, la formación inicial o de grado y la socialización laboral en la práctica escolar), qué razones lo llevaron a optar por la profesión docente; qué motivos lo llevaron a escoger ese centro educativo; concepciones sobre el rol docente, sobre el alumno, sobre la enseñanza. Asimismo en la entrevista les solicitamos la elaboración de dos relatorios sobre una buena clase y sobre un incidente crítico que hubiesen vivido en esos centros.

Los aspectos relevados en las entrevistas a los docentes y a los informantes clave fueron importantes en el proceso de construcción y validación de resultados. Los datos se combinaron con los obtenidos en las observaciones y las narrativas.

Apelar a las narrativas indica ponderar el valor de la forma y la función de los relatos como una vía de acceso al conocimiento permitiendo aproximaciones al ser y al hacer de los sujetos. Jackson (1991) fue uno de los primeros en prestar atención a las narrativas de los docentes sobre su trabajo y a todo lo que ocurre en las aulas haciendo un análisis etnográfico de los pequeños sucesos. McEwan y Egan (1998) destacan la importancia de la narrativa en la investigación cualitativa en educación. Sostienen que la narrativa ayuda a los docentes a darle un sentido nuevo a su vida profesional, contribuyendo a salvar la brecha entre ellos y los que no ejercen la docencia, con la aceptación de la centralidad del relato de historias para el estudio de casos. La función de la narrativa consiste en hacer inteligibles nuestras acciones para nosotros mismos y para los otros, por tanto el discurso narrativo es fundamental en nuestros esfuerzos de comprender la enseñanza y el aprendizaje. La enseñanza en la actualidad apunta asimismo a mirar las prácticas para reconstruirlas.

Al plantearnos la cuestión del potencial de generalización de los resultados obtenidos, somos conscientes de los límites de nuestra investigación. No hemos examinado una amplia gama de casos, por lo tanto no podemos defender la naturaleza general de lo que hemos hallado.

Interludio. Sobre las implicancias con el tema de investigación

De sólito las preguntas de investigación son recurrentes a lo largo de nuestra historia docente, investigativa y personal ${ }^{4}$. Consideramos que analizar la vinculación entre esas preguntas, las cuestiones personales y nuestra trayectoria como docentes, el poder objetivar, contar la historia, ayuda a consolidar las preguntas.

Desde 1979 me dedico exclusivamente al quehacer educativo y desde 1998 en adelante, en la etapa correspondiente a la diversificación según el modelo de Huberman (1999), integro hasta la fecha equipos de dirección en centros educativos que atienden población estudiantil proveniente de contextos desfavorables. De esta experiencia surgen los principales problemas que en primera instancia deseábamos investigar:

1) Las buenas prácticas en contextos desfavorables.

2) El impacto de lo organizacional en centros educativos secundarios públicos que atienden poblaciones pertenecientes a contextos desfavorables. 
En el marco de un año sabático, con el equipo de dirección al que pertenecíamos, realizamos un trabajo escrito con relación a las prácticas de gestión y a factores del estilo institucional que podrían ayudar a logros en los aprendizajes de los estudiantes de esos contextos. Pretendimos sistematizar lo vivido a través de una mirada retrospectiva a partir de la memoria personal y del equipo y de registros escritos. La reflexión sobre las prácticas ${ }^{5}$ llevadas a cabo por dicho equipo constituyó el soporte de ese trabajo. Pero la información disponible era insuficiente para responder interrogantes como: ¿pueden los centros hacer algo para modificar las diferencias sociales originales o su papel se limita a la reproducción y legitimación de las diferencias existentes?

En el presente trabajo el eje de la investigación cambió ya que ahora el foco fue puesto en las prácticas de enseñanza a nivel áulico en centros educativos que trabajan con poblaciones provenientes de contextos de pobreza. Lo esperable sería encontrar prácticas no ortodoxas o no convencionales que resultaran con derivaciones positivas para los estudiantes pero lo interesante podría resultar incluso de lo no esperable.

\section{Acercamiento a los contextos de enseñanza y a sus actores}

\section{Los escenarios y los actores}

Es a partir de la recuperación de testimonios de los protagonistas y de la captación de la especificidad de estos contextos, que hemos podido aproximarnos a la comprensión del sentido que adquieren las buenas prácticas en contextos desfavorables.

Los centros seleccionados son de Ciclo Básico y atienden a jóvenes pertenecientes a sectores sociales pobres. En nuestra investigación hemos conocido a directivos y docentes que reflexionan sobre esas realidades y no se lamentan de la misma, ni se resignan. Esa realidad es asumida desde un punto de vista crítico y constructivo; esto implica una toma de conciencia del contexto en el que están inmersos, un conocimiento de la población estudiantil que nutre al centro, su situación familiar o comunitaria, no para buscar limitaciones sino posibilidades, contando con el compromiso de la comunidad educativa.

\section{Presentación de los centros}

Centro "X" En este centro coexisten dos planes: Reformulación 2006 para $1^{\circ}$ y $2^{\circ}$ y Plan 96 para los grupos de $3^{\circ}$. Cuenta con 754 alumnos distribuidos en dos turnos y 60 docentes siendo 37 de carácter interino. La alta rotación de los docentes incide negativamente en el logro de acuerdos en los criterios de evaluación y pautas de convivencia. El 31, 7\% de profesores tiene una permanencia en el centro de cinco o más años y son los que impulsan fundamentalmente las distintas actividades. El aporte de nuevos docentes es importante en la renovación de ideas pero en algunos casos la falta de permanencia en el centro debilita dicho proceso.

Los resultados académicos correspondientes a diciembre de 2005 y febrero de 2006 evidencian uno de los problemas que la institución no ha logrado revertir a pesar de las acciones implementadas al respecto: la repetición alcanzó el 44,7\% para los primeros años, disminuyendo en el caso de los $2^{\circ}$ a un $29,6 \%$ y para los $3^{\circ}$ se verifica un $40 \%$.

El liceo participa de la red zonal que reúne a varias instituciones que trabajan en temas relacionados con problemáticas que afectan a estos sectores.

Centro "Y" En este centro educativo conviven el Plan '86 Reformulación programática del '93 en los terceros años y la Reformulación 2006 en primero y segundo. Cuenta con 1188 alumnos y 81 profesores. Sus características edilicias no respondían a las necesidades funcionales de un centro educativo, con el agregado que siete salones se orientaban en paralelo a la vía ferroviaria. Esta situación se revirtió, ya que hay un nuevo edificio. 
De acuerdo a los datos brindados por el Censo Nacional de Aprendizajes aplicado a los terceros años del Ciclo Básico (ANEP-MESyFOD, 1999), se caracterizó al centro como de nivel medio bajo. Los datos relevados en los años posteriores confirman que la situación del año 1999 se ve agravada por la incorporación creciente de estudiantes de los estratos más desfavorecidos de la población.

\section{Los docentes}

En cuanto a los profesores, observamos a cuatro mujeres con diferentes edades y experiencia docente. Una de ellas con pocos años de trabajo y las tres restantes podrían ser calificadas de experimentadas (Ropo, 1991) $)^{6}$. Las cuatro trabajan exclusivamente en la docencia en instituciones públicas y privadas. Todas han asistido a cursos de perfeccionamiento docente y dos de ellas estaban estudiando otra carrera.

\section{Primer acercamiento a los datos}

Para realizar el primer análisis del material recolectado en las observaciones de clase y las entrevistas, apelamos a tres conjuntos de dimensiones de análisis: la enseñanza en el aula, la institución y la biografía.

En el primero de ellos, la enseñanza en el aula, tomamos el contenido, el método y la evaluación, derivados de la didáctica clásica e incluidas únicamente con fines exploratorios. Otras dimensiones de análisis tomadas fueron la relación docente - alumno y la motivación expresada por los alumnos. En cuanto a la institución, consideramos la relación del docente con sus colegas, con el centro, con los padres de los estudiantes, con las autoridades y con la comunidad extraliceal. Con relación a las biografías distinguimos la vertiente personal y la profesional, reconociendo que la disociación es meramente artificial a los efectos del análisis, dado que la biografía profesional está permeada por aspectos de la historia del sujeto.

\section{Las recurrencias percibidas nos llevaron a generar cuatro agrupamientos interpretativos:}

Un primer agrupamiento, La complejidad como riqueza surgió del análisis de las entrevistas a los docentes acerca de su visión sobre los estudiantes provenientes de contextos desfavorables, y la relación docente - alumno a partir de las entrevistas y las observaciones de clase. De estos datos emergen las concepciones ${ }^{7}$ docentes acerca de los estudiantes de esos contextos, coincidiendo en caracterizarlos como pobres en diversos sentidos, señalando dificultades varias y percibiendo los límites de su tarea, a la vez que la sienten como un desafío. En éste radica la posibilidad de dar herramientas para el desarrollo personal y social de los estudiantes.

Las docentes caracterizaron a los estudiantes como adolescentes, coincidiendo con construcciones socio - históricas de esta etapa evolutiva desde una mirada adultocéntrica (Perdomo, 1996). Perciben en ellos la necesidad de ser reconocidos y respetados, lo que podría expresar la necesidad de subjetivación, que remitiría a ser reconocido como sujeto por un otro, a la necesidad de filiación, en un momento histórico en que los lazos sociales están fragmentados. Entendemos la producción de subjetividad (Giorgi, 2003) como las formas de construcción de significados, las maneras de percibir, sentir, pensar, conocer, actuar; las modalidades vinculares, los modelos de vida, los estilos de relación con el pasado y el futuro; las formas de concebir la articulación entre individuo y sociedad, todo lo cual se construye a través de prácticas sociales. Éstas operan mediante tres mecanismos: la asignación de roles y lugares, la modelación en tanto contribuye a la identidad y a los estilos de relación con otros y el aprendizaje en sentido amplio. 
El individuo es un sujeto de necesidades que se satisfacen o no en las relaciones vinculares. Las experiencias de satisfacción o frustración son componentes esenciales en la producción de subjetividad y éstas harán a procesos de pertenencia que mantienen al sujeto dentro de los grupos de referencia. Nos preguntamos de qué modo operan los docentes a través de sus prácticas de enseñanza en el proceso de producción de subjetividad en los adolescentes, en el trabajo de filiación simbólica si "(...) necesitan mucho más que la educación disciplinar, y no siempre estamos en condiciones de atenderlos como deberíamos (D2.E)".

Caracterizan como "pobre" el contexto de procedencia de la mayoría de los estudiantes y coinciden en señalar su preocupación por la pobreza lingüística, aludiendo al uso de un código restringido. El lenguaje es un código compartido que nos construye sujetos en relación dialógica, nos introduce en la cultura y tiene una intencionalidad orientada a la comprensión. Nos preguntamos de qué modo enfrentan los docentes las dificultades lingüísticas de los estudiantes en sus prácticas de enseñanza.

Las docentes también coinciden en señalar otras carencias que operan como obstáculos para que los estudiantes aprendan su disciplina: "Dificultades en el acceso a material de corte científico, (...) carencias afectivas y de conductas saludables que impiden la concentración en sus tareas, problemáticas socio-culturales y familiares... (D2.E)". Advierten también obstáculos referentes al desarrollo de la motricidad fina, a lo perceptivo motriz y a lo cognitivo.

Otras dificultades hacen a la falta de proyectos tal como expresa una docente:"(...) Hay mucho de no saber para donde ir, así que nada tiene sentido porque no tenemos una meta. ¿Por qué los jóvenes no tienen una meta? (D1.E)". La falta de proyectos se vincula con el pragmatismo e inmediatismo (Giorgi, 1992) que se presentarían como rasgos adaptativos a las condiciones de vida en contextos de pobreza, en los que prima la lucha por sobrevivir. Pero la incertidumbre que caracteriza el nuevo marco global, genera como efecto el sentir inviable, la formulación de proyectos independientemente de la condición socio-económica. Las bajas perspectivas personales relacionadas con el esfuerzo y el estudio, la dificultad de encontrar una salida laboral lleva al conformismo, al yaísmo y a la desesperanza. Los adolescentes son, hoy un sector especialmente abrumado.

Pero estas dificultades presentan una contracara: “(...) Si bien en la heterogeneidad está la riqueza para nuestra labor, tienen algunas carencias que no podemos solucionar, pero sí darles herramientas para su propio desarrollo e inclusión social (D2.E)".

Del análisis de las entrevistas emerge la inclusión social de la cual sostuvimos que es un concepto en tensión dinámica que se define por el opuesto: el adentro y el afuera. Constatando la exclusión de se define la necesidad de la inclusión en. El término exclusión es multidimensional, no hay acuerdos en cómo definirlo pero sí en lo que sentimos frente a ella, como dice Gentili (2001), el miedo a quedar excluidos nos recuerda la existencia de la exclusión. Afirmamos el carácter político de esta noción que oculta la que emerge de los comentarios de las docentes: la de la pobreza.

Frente a ésta, las docentes ponen en evidencia el conflicto de culturas heterogéneas que se da en las aulas: "[los estudiantes] cuestionan tu lugar de clase media con trabajo, con casa, icon comidas diarias! Y esto es interesante porque no creo en subculturas, creo en culturas diferentes y en los puentes entre una y otra. (D3.E)". Rebellato (1989) sostiene que cuando los profesionales y los sujetos con los cuales se trabaja pertenecen a diferentes culturas, puede producirse un choque ético - cultural, esto es una tensión entre su realidad y la de los otros. Este choque puede asumir diferentes modos de resolución, uno de ellos es a través de la ocultación de las 
diferencias, incurriendo en una práctica demagógica que conduciría a la anulación de su rol profesional; otra modalidad es el despliegue del autoritarismo, imponiendo sus creencias y sus valores, desconociendo al otro. El profesional debe reconocer y analizar las diferencias; esto requiere una actitud autocrítica, reconociendo sus límites y potencialidades. Sólo desde esta actitud es posible el aprendizaje y a través de él la mutua transformación.

Otro agrupamiento, Encontrar un lugar... entre otros, surgió de la convergencia de aspectos indagados en las entrevistas acerca de la autopercepción de los docentes respecto de su rol en el centro en que trabajan, la caracterización del plantel docente del centro y los espacios compartidos con sus colegas. De ellos se deriva que el lugar que tienen en el centro educativo las docentes entrevistadas, supone procesos de descubrimiento o construcción del mismo. Por otro lado perciben que los colectivos docentes están conformados por subgrupos diferenciados. Por último coinciden en la valoración favorable de los aportes de sus colegas.

Un tercer grupo de correspondencias, El desarrollo en curso, emergió del análisis de la historia de formación de las entrevistadas, de los cursos que estaban realizando en el momento de la recolecta de datos y de la razón por la que optaron por la profesión docente. Todas las docentes entrevistadas realizaron cursos de actualización o perfeccionamiento docente en los tres últimos años, coincidiendo también que entre las razones por las que optaron por su profesión, entran en juego docentes de su biografía escolar.

Por último, El conocimiento y la vida cotidiana en las aulas, en el que convergió un conjunto de elementos desde lo referido a la percepción de la asignatura como facilitador del aprendizaje y desde las estrategias de enseñanza que implementan, -aspectos estos derivados de las entrevistas y las observaciones de clase-. Surgieron recurrencias en torno al manejo del error en clase desde lo didáctico y lo moral, al relacionamiento de los contenidos trabajados en clase con otros temas ya dados, a la inclusión de aspectos de la vida cotidiana en el aula, al respeto de los tiempos de aprendizaje, al propiciar la participación y el trabajo colaborativo, lo que nos lleva a pensar en prácticas de enseñanza contextualizada.

\section{La urdimbre}

Haber realizado el análisis de las observaciones de clase, las entrevistas y las narrativas producidas por las profesoras, nos posibilitó generar agrupamientos interpretativos que nos brindaron un mayor acercamiento a la comprensión de nuestro objeto de estudio: las buenas prácticas en contextos desfavorables.

Del análisis de las diferentes fuentes surgen convergencias que nos permiten establecer que existen buenas prácticas de enseñanza que logran entusiasmar a los jóvenes de contextos desfavorables, que se caracterizan por ser contextualizadas, en las que subyace la búsqueda de la comprensividad desde las vertientes epistemológica y moral y se fundamentan en un compromiso docente que trasciende lo disciplinar.

Habiendo analizado las observaciones de clase y las narrativas de lo que las docentes consideraban una práctica acorde a su concepción de buena enseñanza, emergen como recurrencias que los dispositivos de enseñanza que implementan, tienen como característica la flexibilidad evidenciada en la forma que adopta el diálogo en clase tendiente a la participación de los estudiantes, en la atención a los temas emergentes, en el modo de tratar el error, en las actividades propuestas que propician el trabajo colaborativo y en el manejo de los tiempos. 
En la búsqueda de comprender la manera en que estas prácticas se vinculan con la historia personal y profesional de las docentes, del análisis de las entrevistas surge un entramado entre las concepciones que tienen las docentes acerca de la población estudiantil de estos contextos, la autopercepción de su rol en el centro educativo y su historia profesional, que deriva en que las prácticas de enseñanza con estas poblaciones se asumen como un desafío. Éste surge del encuentro con una realidad social nueva de la que emergen nuevas poblaciones estudiantiles; éstas son concebidas por las docentes como pobres, considerando la multiplicidad de la pobreza. Conciben su rol docente como un compromiso personal y profesional, enmarcado en la profesionalidad ética.

En cuanto a las historias de formación, las concepciones que prevalecen en las docentes se inscriben en lo institucionalizado y la historia profesional queda circunscripta a las instituciones en las que se desempeñan actualmente.

No pudimos establecer convergencias entre la historia personal y profesional de las docentes. Solamente recuperamos la historia de formación entramada con la historia personal a partir del relato de una docente, quien mediante un proceso reflexivo significó lo vivido. De su narrativa emerge una concepción amplia de formación que comprende su pasaje por diferentes instituciones educativas formales y no formales en las que asumió diferentes roles, reconociendo "marcas" que algunos profesores dejaron en ella. La característica compartida por esos docentes era que en sus clases mostraban otras formas de acceso al conocimiento; uno de ellos podría asimilarse a la categoría de docente memorable. Señaló experiencias de trabajo con poblaciones infantiles y adolescentes pobres que le permitieron concebir la ética como una ética de vida que trasciende lo docente y que se funda en reconocer al otro en dignidad.

En base a este nuevo nivel de análisis generamos otros agrupamientos interpretativos:

- La novedad del encuentro con los nuevos.

- Utilizando viejas herramientas con nuevos sentidos.

- Pensando la novedad en colectivo

La novedad del encuentro con los nuevos

Del análisis efectuado a partir de las distintas fuentes, surge un entramado de las concepciones que tienen las docentes acerca de la población estudiantil de estos contextos, el vínculo que establecen con los estudiantes y aspectos relativos a la autopercepción de su rol en el centro educativo y su historia profesional. Todas las docentes conciben las prácticas de enseñanza con estas poblaciones como un desafío que se genera del encuentro con una realidad social nueva de la que emergen nuevas poblaciones estudiantiles, concebidas por las docentes como pobres en diversos sentidos. Conciben su rol docente como un compromiso personal y profesional, enmarcado en la profesionalidad ética.

De acuerdo a lo analizado, las docentes perciben que la población estudiantil presenta carencias y dificultades de diferente índole: códigos lingüísticos restringidos; dificultades que surgen en el desarrollo de la motricidad o lo cognitivo, carencias afectivas y de conductas saludables; dificultades en el acceso a materiales de trabajo y estudio, enmarcados todos en problemáticas socio culturales y familiares, que dificultan la construcción de proyectos.

Con relación al trabajo con esta población y relacionado con el punto anteriormente planteado, coinciden en señalar dificultades varias, admitiendo los límites de su tarea, a la vez que reconocen que en la heterogeneidad de la población estudiantil radica la riqueza de la misma, sintiéndola como un desafío. En éste radica la posibilidad de dar herramientas para el desarrollo personal y social de los estudiantes, lo cual se presenta como la contracara de las dificultades, ya que se enmarca en el horizonte de lo posible, como posibilidad esperanzadora. 
Consideramos importante la valoración de la dificultad como desafío; las docentes no le atribuyen a la dificultad el carácter de irreversible ya que esto último bloquearía la acción. Al tomar la condición de contexto de pobreza como algo que se opone a lo conocido y sobre lo cual tienen que aprender, lo perciben como movilizador y provocador de acciones que posibilitan cambios.

Ese desafío también lo asumen en el relacionamiento que establecen con los estudiantes, tal como fue observado en las clases, en un vínculo cercano que mantiene la asimetría, ejercen la autoridad sin perder la cercanía, invitan a la participación desde una actitud de escucha atenta. La invitación constante a la participación y compromiso activo con el trabajo y a través de él con el conocimiento, trasunta la confianza de las docentes en las posibilidades de aprendizaje de todos los alumnos, brindándoles también atención personalizada y fomentando la autonomía.

El modo de relación que establecen los docentes, basado en el respeto, el afecto y la confianza genera un clima de aula favorecedor de los aprendizajes. En las distintas clases observamos una actitud entusiasta en los docentes y notas de humor sin que se viera afectado el orden y la disciplina.

El vínculo que establecen parecería estar fundado en el reconocimiento de las múltiples necesidades de los estudiantes. Todos somos sujetos de necesidades que se satisfacen o no en relaciones vinculares; la satisfacción o no de las mismas opera en la producción de subjetividad, condicionando los procesos de filiación de los sujetos con respecto a los grupos de pertenencia. Generar vínculos de sostén desde el reconocimiento del otro como sujeto, favorece la construcción de subjetividad y los procesos de filiación.

Las cuatro docentes pertenecen a un contexto socio-económico-cultural diferente al de las poblaciones estudiantiles con las que trabajan. En el encuentro de estas dos culturas, podría producirse un choque ético - cultural, una tensión entre la realidad de las docentes y la de los estudiantes. En el caso de todas las docentes, las diferencias no se ocultaron, sino que se admitieron como lugar de tensión, choque y cuestionamiento y es a partir de este reconocimiento que enmarcan sus propuestas de enseñanza. Reconocimiento que surge de señalar sus propias potencialidades pero también sus límites. Sólo desde esta actitud es posible el aprendizaje y a través de él, la mutua transformación.

En este sentido, tal como emerge del análisis de todas las entrevistas, el relacionamiento que establecen con los estudiantes es capaz de afectarlas de algún modo. Este reconocimiento del otro como sujeto que un vínculo es capaz de afectar, se relaciona con la posibilidad de transformarse a través del mismo que implica a los docentes y a los estudiantes. Las cuatro docentes coinciden en señalar elementos que relacionan la enseñanza, el aprendizaje y lo vincular, desde una mirada que involucra un aspecto del que poco se habla en la enseñanza: el amor.

Utilizando viejas herramientas con nuevos sentidos

A través del análisis de las observaciones de clase y de las narrativas de lo que las docentes consideraban una práctica acorde a su concepción de buena enseñanza, tratamos de recuperar los distintos elementos que caracterizan las prácticas áulicas en contextos desfavorables teniendo presente, por otra parte, las difíciles y complejas condiciones en que se llevan a cabo.

La consideración del contexto en el que se inscriben las prácticas de enseñanza y las condiciones que las enmarcan, llevaron a las docentes a implementar dispositivos de acción que tienen como característica el ser flexibles. La contextualización y la flexibilidad de las prácticas se entretejen en estrategias que pretenden habilitar situaciones de aprendizaje tendientes a la comprensividad. 
Una de las recurrencias encontradas es precisamente, la preocupación por la comprensividad: aparece dada en el diálogo constructivo en el que se utiliza la pregunta como guía del conocimiento; las preguntas son de carácter conciso y actúan como articuladoras entre lo que el estudiante conoce y el nuevo contenido oponiéndose al método explicativo tradicional. Otro articulador posible que surge del análisis de las observaciones es que las docentes apelan al lenguaje coloquial sin dejar de lado el lenguaje técnico de la asignatura, invocando en ocasiones, expresiones que surgen de la televisión.

Uno de los desafíos que afrontan es que lo nuevo a aprender se vincule con temas ya sabidos integrándose en la estructura de conocimientos que el alumno posee tratando de establecer relaciones. Identifican temas y conceptos relacionándolos entre sí, permitiendo establecer una trama de relaciones. La relación con sentido da cuenta de que lo nuevo aprendido no quedó aislado ni se perdió.

En el contexto áulico, las docentes atendieron los emergentes considerándolos como oportunidad de favorecer los procesos de comprensión más allá de lo estrictamente disciplinar y dando la posibilidad de generar otros sentidos. Lo inesperado o lo incierto también es contemplado. En este sentido, del análisis de las observaciones surge que las situaciones que implicaron la ruptura de alguna pauta de conducta, fueron señaladas sin sanción, apelando a la indicación de pautas adecuadas al contexto áulico, a veces desde el humor.

En el marco de lo inesperado e incierto, emerge en forma particular el error. De las observaciones de clase se desprende que las docentes transforman el error en una oportunidad educativa en el plano didáctico y moral. Integran a la clase el tratamiento activo del error, también incluyen aspectos de la socialización y además les brindan a los alumnos la experiencia cotidiana de un trabajo intelectual auténtico. En la oralidad, el error no es sancionado, sino que se devuelve en forma de pregunta precisando los conceptos.

Flexibilidad y búsqueda de la comprensividad se ponen de manifiesto en la forma que adopta el diálogo y en las propuestas de actividades que favorecen la participación y el trabajo colaborativo.

A punto de partida del análisis de las observaciones, las cuatro docentes propiciaron la participación de los estudiantes en la adquisición del conocimiento, relacionando los contenidos trabajados con otros ya dados, incluyendo aspectos de la vida cotidiana en el aula, proponiendo tareas en equipo que se inscriben en la concepción del aprendizaje colaborativo. El punto de partida de las docentes para estructurar las estrategias de enseñanza es la intención de apoyar el desarrollo personal de cada estudiante, por lo cual hacen un manejo flexible de los tiempos y respetan los ritmos de aprendizaje.

Estimular la participación grupal y propiciar el trabajo colaborativo, no impidió que las docentes brindaran atención personalizada a los alumnos, con una mirada atenta a cada estudiante, en especial a aquellos que no se involucraban con el trabajo, mirada atenta aunque no sancionadora sino de cuidado, en el mejor sentido de la palabra. Estas acciones docentes evidencian la confianza en las posibilidades de aprendizaje de los estudiantes y se relacionan con el sostén que las docentes hicieron con respecto a las intervenciones de los estudiantes, cuando las reafirmaban con expresiones de aprobación.

Otras recurrencias surgen del análisis de las entrevistas y las observaciones de clase al considerar un conjunto de elementos desde las concepciones docentes acerca de los facilitadores u obstaculizadores para que los estudiantes aprendan su disciplina y desde las estrategias de enseñanza que implementan. Surgen recurrencias en cuanto al carácter provisional del 
conocimiento disciplinar, la concepción de la disciplina como un facilitador del aprendizaje y el acceso cotidiano de los estudiantes a los objetos de conocimiento fuera del ámbito de la enseñanza formal. Convergen también el respeto a la asignatura o al docente, dado por la pasión por el pensamiento y el conocimiento, que generaría a su vez, otros sentidos en aquellos a quienes se enseña.

Desde todo lo enunciado en este apartado, emerge el carácter transformador de estas prácticas de enseñanza que apuntan a la comprensividad desde los contextos en que tienen lugar.

\section{Pensando la novedad en colectivo}

Del análisis de las razones que llevaron a las docentes a optar por ese centro educativo, la autopercepción de los docentes respecto de su rol en el centro, la caracterización del plantel docente del centro y los espacios compartidos con sus colegas y los facilitadores y obstáculos percibidos en el centro para la enseñanza de su disciplina, se deriva que el lugar que tienen en el centro supone procesos de descubrimiento o construcción del mismo en colectivos docentes en los que reconocen subgrupos diferenciados y a los que valoran favorablemente. Conciben su rol docente como un compromiso personal y profesional, enmarcado en la profesionalidad ética.

Reconocen, a su vez, la coordinación como un espacio privilegiado para pensar, siendo un espacio de trabajo colectivo donde se toman decisiones y se da sentido a la tarea docente. En relación con el centro surge la consideración que la entidad colectiva es un facilitador de la enseñanza.

Los elementos que se evidencian con más fuerza se relacionan con la afectividad, el sentido de pertenencia y la posibilidad de pensar sus prácticas de enseñanza con otros. Lo afectivo aparece en las razones que incidieron en la elección del centro. Con respecto a la autopercepción del rol, emerge la noción de construir un lugar reconociendo un papel activo que refuerza el componente afectivo. Estos elementos coadyuvan a cimentar el sentido de pertenencia que está presente en las cuatro docentes.

Otro grupo de correspondencias se estableció tomando en cuenta al desarrollo profesional: todas las docentes realizaron cursos de actualización o perfeccionamiento docente en los tres últimos años y valoran la importancia de la formación (Instituto de Profesores Artigas, cursos de actualización, etcétera) que posibilitarían la mejora de sus prácticas de enseñanza y la colectivización del conocimiento desde la vertiente disciplinar, la didáctica de la disciplina o la que se deriva de contar con herramientas teóricas para trabajar con esos estudiantes.

Concuerdan en la posibilidad de multiplicar lo aprendido (a los estudiantes y a los colegas) y de pensar con otros, que coincide también con las razones por las que optaron por su profesión. Multiplicar lo aprendido y pensar con otros, son aspectos que hablan de la construcción de una profesionalidad ética. Esta comprende la generosidad con el conocimiento y la socialización de saberes.

En momentos en que la razón instrumental reifica los saberes, convirtiendo el conocimiento en mercancías, la profesionalidad ética busca transformar el conocimiento en bien común, apuntando de esta forma a la democratización del mismo, al hacerlo público, dentro de los diferentes colectivos, esto hace de la ética un acto político.

Hablamos de profesionalidad ética que se construye con otros y de un conocimiento que al hacerse colectivo, abre la posibilidad de pensar sobre él en el marco de las prácticas educativas, lo que lleva a una dialectización en procesos de acción y pensamiento-reflexión. 
La profesionalidad ética emerge también de las razones por las que las docentes optaron por la profesión, lo que remitiría a una dimensión ontológica que incluiría aspectos de su biografía (personal y escolar) en la que algunos docentes operaron como ejemplos o contra-ejemplos. Con relación al porqué de la opción por la docencia emerge que las docentes consideran que ser verdadero docente trasciende lo estrictamente disciplinar, apuntan a la preocupación moral acerca de la manera en que cada estudiante logra construirse como persona de acuerdo a sus circunstancias vitales, manifestando también el placer de trabajar con los jóvenes a través de su disciplina.

Por lo anteriormente desarrollado podríamos pensar que en la profesionalidad ética se vinculan dimensiones epistemológicas y morales.

\section{Conclusiones}

En lo personal, quisiéramos dejar constancia que llegamos a este tema por una preocupación esencial: aprender a mirar con otros ojos las buenas prácticas en contextos desfavorables.

Hicimos un análisis del contexto en que se inscriben esas prácticas ya que es el ámbito en que se significan y esto permitiría comprenderlas como prácticas sociales.

Los docentes y la escuela como institución enfrentan desafíos a partir de las transformaciones ocurridas a nivel estatal y social como: la situación de pobreza de la mayoría de los estudiantes que, lejos de homogeneizar, produce la heterogeneidad de la población estudiantil; las dificultades diversas a nivel cognitivo; subjetividades diferentes a las que propone el centro educativo.

Hecho el análisis de los datos relevados de las diferentes fuentes de información y realizada la triangulación correspondiente entre los mismos, estamos en condiciones de concluir que las docentes estudiadas reconocen el contexto de procedencia de los estudiantes admitiendo que la riqueza de su tarea está en la heterogeneidad de la población estudiantil que atienden, toman en cuenta lo plural y lo diverso de esos sujetos que aportan su potencial, y fortalecen los vínculos desde donde poder gestar proyectos o buscar puertas de "acceso a la cultura".

Admiten que el trabajo con esta población les resulta desafiante, pero expresan la certeza de poder dar herramientas, de la posibilidad. Postulan una ética del reconocimiento que se construye dialógicamente y que contribuye a cambiar sus concepciones y su relación con los demás. Estas docentes muestran su interés por estos jóvenes como personas, facilitando su construcción como sujetos, asignando importancia a la dimensión emocional vinculada a la relación educativa.

La propia complejidad del contexto las llevó a diseñar propuestas que se adecuaran al mismo generando estrategias metodológicas que favorecieran los procesos cognitivos en los estudiantes. Las prácticas de enseñanza en medio de la compleja realidad de las pobrezas, constituyen un desafío para poder transformar los obstáculos en oportunidades, encontrar caminos o posibilidades para descubrir lo que cada uno posee en tanto es.

Estas buenas prácticas de enseñanza suponen un quehacer comprometido con el contexto.

Lo sostenido discursivamente por las docentes es coherente con lo desplegado en las prácticas de aula y en otras actividades en el centro educativo, lo que nos lleva a considerar como un posible hallazgo el compromiso docente con la enseñanza, con esta población estudiantil y con los centros desde una mirada que va más allá de la enseñanza disciplinar. 
Las docentes evidencian un alto nivel de compromiso con su trabajo, éste les resulta gratificante y esto hace que establezcan un nivel de relación afectiva con los estudiantes, con los pares, el centro y su labor pedagógica. Este compromiso les permite afrontar los obstáculos que encuentran en su accionar profesional que se entrelaza con las expectativas que tienen de sus alumnos, intentando afectarlos positiva e integralmente.

En todos los casos observamos una dedicación a la tarea y una actitud generosa con el conocimiento y con el otro, destacándose el deseo de perfeccionamiento y la capacidad de llevar adelante su trabajo con entusiasmo.

Las docentes y los directivos de los centros en los que trabajan orientan su tarea hacia el encuentro de caminos para apoyar el desarrollo personal de cada estudiante, que pareciera ser centro de sus preocupaciones.

Las docentes de la muestra presentan algunas características comunes: se expresan con claridad, estimulan o favorecen la participación de los estudiantes, tienen dominio de los contenidos de la materia que enseñan, planifican su trabajo de manera flexible y apoyan a los alumnos con dificultades. Su forma peculiar de hacer se caracteriza por el respeto, la cordialidad y a menudo expresiones de afectividad. Logran la empatía necesaria para las prácticas de enseñanza, empatía que está basada en procesos de identificación, de reconocimiento del otro como par en igualdad. Unido al compromiso y al agrado que manifiestan podemos reconocer la configuración de un colectivo donde cada uno se siente perteneciente al mismo.

Señalamos anteriormente que en relación con el centro surge la consideración que la entidad colectiva es un facilitador de la enseñanza. La conformación de la entidad colectiva se une, al parecer fructíferamente, con la existencia del otro como persona. Sin embargo el sistema de educación media no ha logrado implementar mecanismos para evitar la alta rotación docente impidiendo la estabilidad y el concomitante desarrollo del sentido de pertenencia a los centros. Nos preguntamos si no sería oportuno buscar mecanismos que facilitaran la permanencia en los centros y habilitaran la posibilidad de formar equipos de trabajo.

Entidad colectiva y existencia del otro como persona son aspectos que aparecen necesariamente unidos el uno al otro. No se puede tener espíritu colectivo si cada actor educativo no se siente tomado en consideración y útil al colectivo. No pueden desarrollarse relaciones humanas de calidez y afecto, sin una organización viva y que busca lo colectivo.

Las docentes de la muestra al caracterizar al plantel docente de los centros en que trabajan señalaban subgrupos con actitudes diferentes ante la misma problemática pero valoran favorablemente esta diversidad. Trabajar con otros, encontrarse con otros en el intercambio de ideas y experiencias es una forma de hacer frente a la nueva realidad que encuentran en las aulas.

Arriesgamos como interpretación que quizás las docentes, para enfrentarse a lo novedoso, buscan al grupo intentando superar el aislamiento, sintiendo tal vez la necesidad de no hacerlo solas. Cuando nos referimos al grupo, en aras de constituir equipos pensamos que toman en cuenta a otros en el proceso de construcción de su propia identidad que no es una identidad en solitario sino una construcción con otros. Esa apelación al colectivo agrega otro valor ético importante que es percibir al otro en igualdad desde una participación activa. Esos valores se expresan en el terreno de las relaciones. 
Señalábamos anteriormente que las docentes de la muestra asumen como un desafío enfrentarse a los nuevos y a lo nuevo, no proceden de ese contexto pero optan por esas poblaciones con un compromiso ético y político.

La profesionalidad ética implica: considerar la docencia como un compromiso moral y ético para el que la desempeña, asumiendo como desafío que los alumnos logren desarrollarse como personas a través del aprendizaje; supone un compromiso con la comunidad social en que se realiza la práctica y también la competencia profesional. Esta última implica disponer de un capital de conocimientos disponibles, que se diversifica al ser compartido en el colectivo; el poder reflexionar críticamente sobre la práctica y finalmente deben estar presentes la complicidad, el afecto y la emotividad, hacer la tarea con placer y sacar alegría de ella, en forma personal y colectiva. El docente que reflexiona construye su profesionalidad.

Cabe señalar que, como en todo trabajo de investigación a la vez que se obtienen respuestas a las interrogantes que determinaron su realización, también se originan nuevas preguntas e inquietudes que posibilitarán nuevos análisis en el futuro. En primera instancia, el abordaje de la presente investigación está circunscrito a un tiempo, a una sociedad y a una zona de Montevideo determinados, con esto queremos señalar que los hallazgos son válidos para el actual contexto de investigación.

Queremos aclarar el significado que este trabajo tuvo para nosotros. Sabemos que los docentes son el recurso más importante en la enseñanza. De sólito han sido considerados sólo como intermediarios del saber curricular, con competencia en lo disciplinar. Se sostiene que los enseñantes tienen experiencia pero no el "saber", el conocimiento lo hacen los externos a la práctica. No obstante las docentes estudiadas lograron elaborar un lenguaje propio, legitimado entre sus propios pares. Su autoridad proviene del reconocimiento de su autoría desde la práctica.

Las docentes elaboraron sus propuestas de acción tomando en cuenta la complejidad del contexto. La variedad de las actividades, la originalidad de las propuestas para evitar la rutinización desafiando los procesos cognitivos y el estímulo para que cada alumno se involucre de manera responsable en la concreción, no tiene recetas y forma parte del oficio de enseñar.

Decíamos al comienzo que si lo no conocido es un obstáculo que dificulta el pasaje, hay que realizar un proceso de visibilización del mismo, reconocerlo como tal para poder transformarlo en problema y luego, en un segundo momento, buscar los modos de superarlo: se exploran estrategias, se diversifican acciones. La condición de adverso origina búsquedas y nuevas exploraciones.

Esto es parte del desafío de enfrentarse a lo nuevo y a los nuevos en un mundo caracterizado por su complejidad, que genera incertidumbre y que requiere con urgencia el diseño de nuevas herramientas, que bien pudieran ser viejas herramientas pero empleadas con nuevos sentidos, desde una actitud que dé "lugar a la presencia del otro" (Eco).

A modo de cierre, consideramos que las prácticas de enseñanza en medio de la compleja realidad de las pobrezas es uno de los mayores desafíos para poder convertir las dificultades en oportunidades, las pobrezas en la posibilidad de descubrir lo que cada uno posee en tanto es. Tal vez se trate, citando a Camus, de alimentar "un hambre más esencial para el niño que para el hombre que es el hambre de descubrir" porque como dice Proust: "El verdadero viaje de descubrimiento no consiste en buscar nuevos paisajes sino en tener nuevos ojos". 


\section{Bibliografía}

ANEP - UMNRE. 1999. Estudio de los factores institucionales y pedagógicos que inciden en los aprendizajes en las escuelas primarias de contextos sociales desfavorecidos en el Uruguay. Montevideo: ANEP.

Barbier, J.M.; Galatanu, O. (comp.) 2000. Relación establecida, sentido construido, significación dada. París: P.U.F.

Bruner, J. 1997. La educación, puerta de la cultura. Madrid: Visor.

Bruner, J. 1988. Realidad mental, mundos posibles. Barcelona: Gedisa.

Camilloni, A. De herencias, deudas y legados. Una introducción a las corrientes actuales de la didáctica. En: Camilloni, A. et. al. 1997. Corrientes didácticas contemporáneas. Buenos Aires: Paidós. pp. 17-39.

Carr, W.; Kemmis, S. 1988. Teoría crítica de la enseñanza. Barcelona: Martínez Roca.

Carr, W. 1999. Hacia una ciencia crítica de la educación. Barcelona: Alertes.

Edelstein, G. 1996. Un capítulo pendiente: el método en el debate didáctico contemporáneo. En: CAMILLONI, A. et. al. Corrientes didácticas contemporáneas. Buenos Aires: Paidós. pp. 75 - 89.

CEPAL. 1990. Enseñanza primaria y ciclo básico de educación media en el Uruguay. Montevideo: CEPAL.

CEPAL. 1991. ¿Qué aprenden y quiénes aprenden en las escuelas del Uruguay? Los contextos sociales e institucionales de éxitos y fracasos. Montevideo: CEPAL.

CEPAL. 1992. ¿Aprenden los estudiantes en el Ciclo Básico de Educación Media? Montevideo: ANEP-CODICEN-CEPAL.

CEPAL. 1994. Los bachilleres uruguayos: quiénes son, qué aprendieron y qué opinan. Montevideo: ANEP-CODICEN-CEPAL.

Eisner, E. 1998. Cognición y currículum. Buenos Aires. Amorrortu.

Elisner, E. 1998. El ojo ilustrado. Indagación cualitativa y mejora de la práctica educativa. Barcelona: Paidós.

Filgueira, C. 2002. Sobre las revoluciones ocultas: la familia en Uruguay (versión preliminar). Montevideo: CEPAL.

Frega, A. L.; Kovadloff, S.; Tedesco, J.C. 2004. Repensando la cultura en términos de educación. [online] [citado 24 Abril 2007]. Disponible en Internet:

http://www.acaedu.edu.ar/espanol/paginas/novedades/foro.htm

Gentili, Pablo. 2001. La exclusión y la escuela: el apartheid educativo como política de ocultamiento. En: revista Docencia, Año VI (15).

Katzman, R. 1996. Marginalidad e integración social en Uruguay. Montevideo: CEPAL.

Katzman, R. 2001. Seducidos y abandonados: el aislamiento social de los pobres urbanos. En: Revista de la CEPAL (75).

Laurnaga, $M^{a}$ E. "Inclusión social y exclusión. Reflexiones para compartir". Curso de Sensibilización a docentes para trabajar en contextos de vulnerabilidad social [CD-ROM]. Montevideo: CECAP - ANEP -CODICEN, 2005. 
Litwin, E. 1997. Las configuraciones didácticas. Una nueva agenda para la enseñanza superior. Buenos Aires. Paidós.

Mallo, S. 2004. El currículo y el papel de los docentes en el contexto actual de la sociedad de mercado. Reflexiones para un debate. Congreso de Educación. Montevideo: Instituto Crandon.

MAX - Neef, M. 1984. La economía descalza. Señales desde el Mundo Invisible. Buenos Aires: Editorial Nordan - Comunidad.

MC Ewan, H.; EGAN, K. 1998. La narrativa en la enseñanza, el aprendizaje y la investigación. Buenos Aires: Amorrortu.

Memfod. ANEP-CODICEN. 2003. CUADERNOS DE TRABAJO. Serie aportes para la universalización del Ciclo Básico de Educación Media $N^{\circ}$ 1. Programa de Modernización de la Educación Media y la Formación Docente. Montevideo: MEMFOD. ANEP-CODICEN.

Mercer, N. 1997. La construcción guiada del conocimiento. Barcelona: Paidós.

MES y FOD. ANEP - CODICEN. 1999. Censo Nacional de Aprendizajes en terceros años del ciclo básico. "Primera comunicación de resultados". Montevideo: ANEP.

MES y FOD y UTU/BID. 2000. Análisis del perfil de las familias de los estudiantes. Segunda comunicación. Montevideo: ANEP - CODICEN.

MES y FOD y UTU/BID. 2000. Los aprendizajes y su relación con los factores institucionales y de gestión pedagógica. Sexta comunicación. Montevideo: ANEP-CODICEN.

Pedernera, L. 2005. Educación y exclusión social. En Voces. IX (19).

Pineaul, P. 2006. De la seducción en el acto pedagógico. Marcas en la cultura argentina. En: FRIGERIO, G. - DIKER, G. (comps.), Educar: figuras y efectos del amor. Buenos Aires: Del estante editorial.

Ravela, P. 1993. Escuelas productoras de conocimientos en los contextos socioculturales más desfavorecidos. Montevideo: CEPAL.

Rivero, J. 1999. Educación y exclusión en América Latina. Reformas en tiempos de globalización. Madrid: Miño y Dávila editores.

Ropo, E. Diferencias en la enseñanza de docentes de inglés: expertos y principiantes. En Carretero, M. (comp.) 1991. Procesos de enseñanza y aprendizaje. Buenos Aires: Aique.

Sarason, S. 2002. La enseñanza como arte de representación. Buenos Aires: Amorrortu.

Sierra Bravo, R. 1988. Técnicas de investigación social. Teoría y ejercicios”. Madrid. Paraninfo.

Sierra Bravo, R. 1984. Ciencias sociales. Epistemología, Lógica y Metodología. Teoría y ejercicios. Madrid: Paraninfo.

Sirvent, Ma T. Múltiples pobrezas, violencia y educación. En: Izaguirre, I. 1998. (comp.), Violencia social y derechos humanos. Buenos Aires: Eudeba.

Taylor S. J.; BOGDAN, R. 1996. Introducción a los métodos cualitativos de investigación. Buenos Aires: Paidós.

UNICEF. 2003. Educación, derechos y participación. Aportes para la reflexión sobre la enseñanza media en el Uruguay. Montevideo: UNICEF. 
UNICEF. 2003. El trabajo infantil y adolescente en Uruguay y su impacto sobre la educación. Análisis de la situación en la década pasada y el presente. Montevideo: UNICEF.

Valles, M. 1997. Técnicas cualitativas de investigación social. Reflexión metodológica y práctica profesional. Madrid.: Editorial Síntesis.

Wainerman, C.; SAUTU, R. 2000. La trastienda de la investigación. Buenos Aires: Lumiere.

Wittrock, M. 1989. La investigación de la enseñanza, I. Enfoques, teorías y métodos. Madrid: Paidós Educador.

Zaffaroni, C. Familia - escuela - comunidad. Curso de actualización a docentes para trabajar en contextos de vulnerabilidad social. Montevideo: CECAP - ANEP - CODICEN.

Woods, P. 1998. Investigar el arte de la enseñanza. El uso de la etnografía en la educación. Barcelona: Paidós.

\section{Notas}

${ }^{1}$ Langón, M. IDENTIDAD (estudio de un concepto en el contexto actual). [online] [citado 8/ 02/07]. Disponible en Internet: ipes.anep.edu.uy/documentos/curso_dir_07/materiales/identidad.pdf

${ }^{2}$ Barbier, J. M.; Galatanu, O. (Comps.). 2000. Relación establecida, sentido construido, significación dada: "Las representaciones vinculadas a una experiencia no se refieren, en efecto a la actividad, sino igualmente al sujeto comprometido en la actividad y a las relaciones en el entorno. La construcción de sentido comprende pues igualmente una puesta en relación de las imágenes de sí mismo como sujeto actuante correspondientes a las experiencias anteriores y a las experiencias nuevas". (p.7)

${ }^{3}$ Carr, W. 1999. Hacia una ciencia crítica de la educación. "Todas las teorías educativas son teoría de la teoría y de la práctica. (...) La teoría es el marco de pensamiento que guía la práctica, teniendo en cuenta esto la teoría estaría conformada por tradiciones que guían nuestra práctica, siendo éste un modo heredado y aceptado de pensar. Viendo estas acepciones de la teoría observamos que la última está relacionada de forma estrecha con las prácticas, pero aquí está el desafío de una teorización desde la práctica, ya que se debe introducir una valoración crítica de los elementos prevalecientes a las mismas tales como conceptos, suposiciones y valores". (p.72)

${ }^{4}$ Peter Woods (1998:15) sostiene que:

"En ocasiones las personas que investigan lo hacen para descubrir cosas sobre sí mismas. Esto no quiere decir que se trate de autoindulgencia, sino que es principalmente por medio de uno mismo como se llega a conocer el mundo. Y a la inversa, los descubrimientos que hacemos se revierten sobre nuestra persona, que vuelve a reflejarlos en la investigación, y así sucesivamente".

5"El profesorado, como todo adulto que aprende necesita partir de la práctica para volver a la práctica en un proceso de cambio..." Imbernón. (1994:81). 
${ }^{6}$ El carácter de experimentado se basa en la pericia, la cual se relaciona con habilidades técnicas, el análisis crítico de las situaciones, acciones deliberadas eficaces basada en estructuras de conocimiento (ROPO, 1991).

${ }^{7}$ Entendemos por concepciones aquellos esquemas que el sujeto construye para dar inteligibilidad a lo real y que operan como un marco referencial desde el cual percibe, actúa y da sentido a la realidad, estando atravesadas por el sentido común (Meirieu, 1997:54).

Las concepciones influyen en las prácticas (Bruner, 1997) y en las decisiones que toman acerca de ellas, involucrando dimensiones intelectuales y éticas.

*El Artículo se enmarca en la tesis de maestría de la autora, dirigida por la Dra.Adriana Chiancone. Año 2008.

**Master en Educación, Universidad ORT Uruguay. Diploma en Educación, Universidad ORT Uruguay. Profesora de Educación Media - Especialidad Historia, Instituto de Profesores Artigas. Directora de Centro, Consejo de Educación Secundaria. 